\title{
Understanding Particle Loss Rates in the Muon g-2 Experiment Storage Ring
}

\author{
Mike Syphers
}

Northern Illinois University

Fermi National Accelerator Laboratory 


\section{The Muon g-2 Experiment}

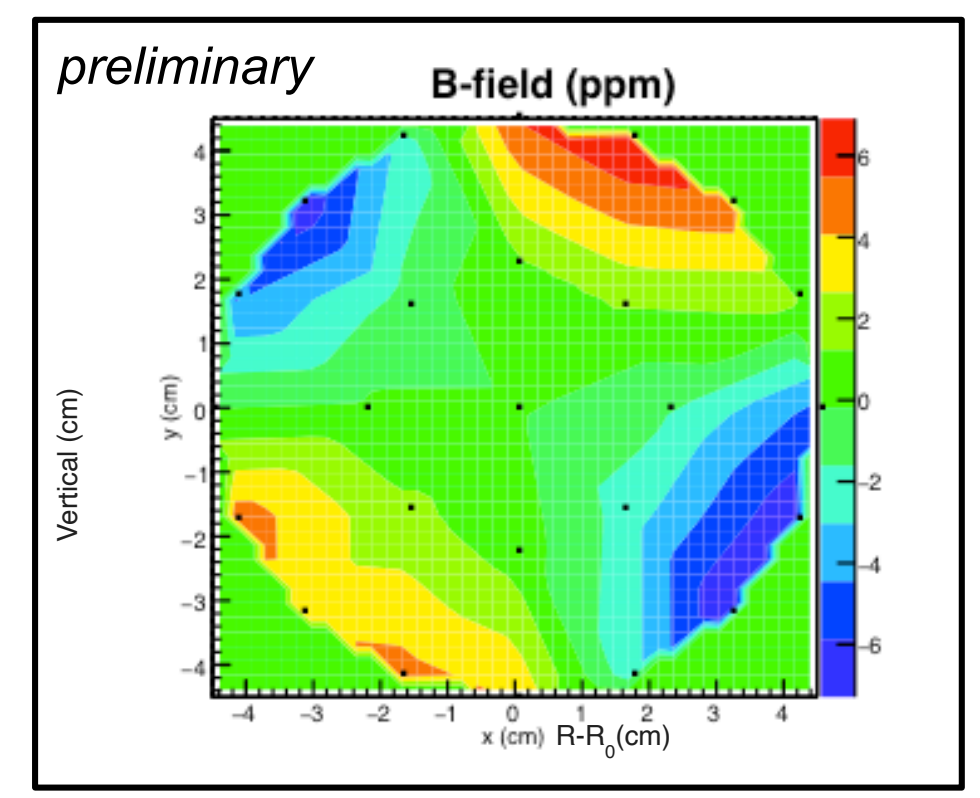

highly uniform $B$ field

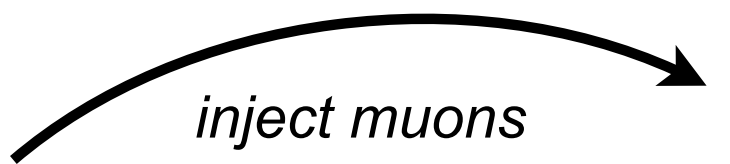

ideal: highly polarized, with spin aligned with momentum

detector rates will "wiggle" with the anomalous precession frequency $\propto g-2$
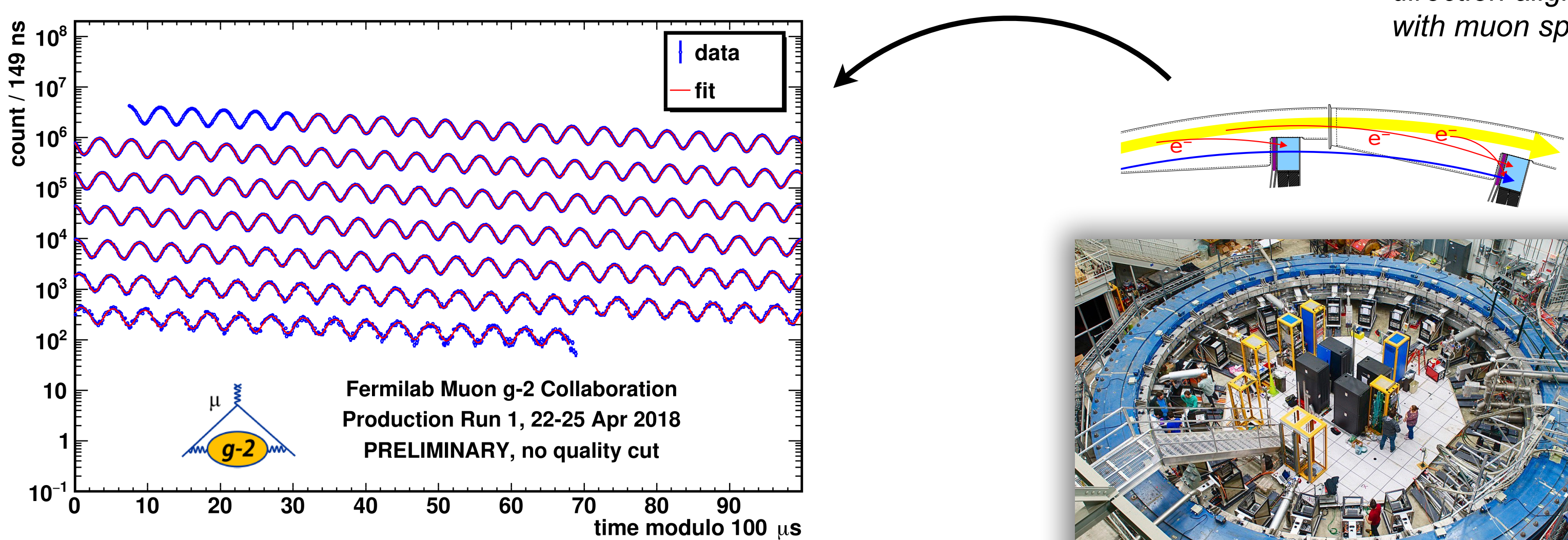

detect positrons from muon decay; high energy $e^{+}$ direction aligned with muon spin magnetic moment

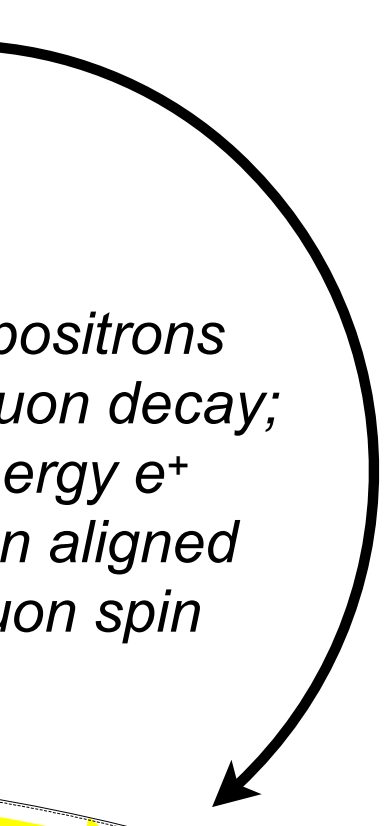
g-2 ma

$$
N(t)=N_{0} e^{-t / \tau_{\mu}}\left[1+A \cos \left(\omega_{a} t+\phi_{0}\right)\right]
$$

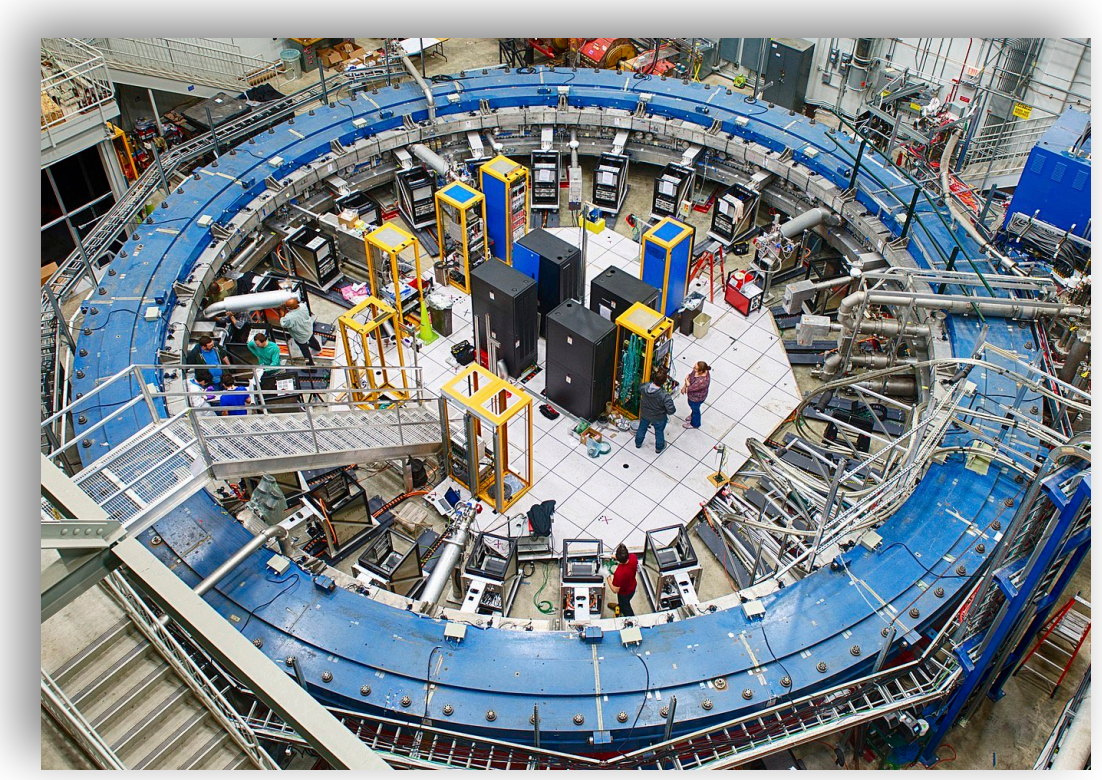




\section{Observed Loss Rates}

Northern Illinois University

- Following the initial injection losses (first 10-20 turns), and factoring out the muon decay process, a slow loss of muons prior to decay continues to persist, and exponentially reduces during the measurement

» a "triple coincidence" in adjacent calorimeters indicates a direct muon hit

» observed in BNL version of the experiment, as well as at FNAL

$B N L$ - PRD 73, 072003 (2006)

FINAL REPORT OF THE E821 MUON ANOMALOUS ...

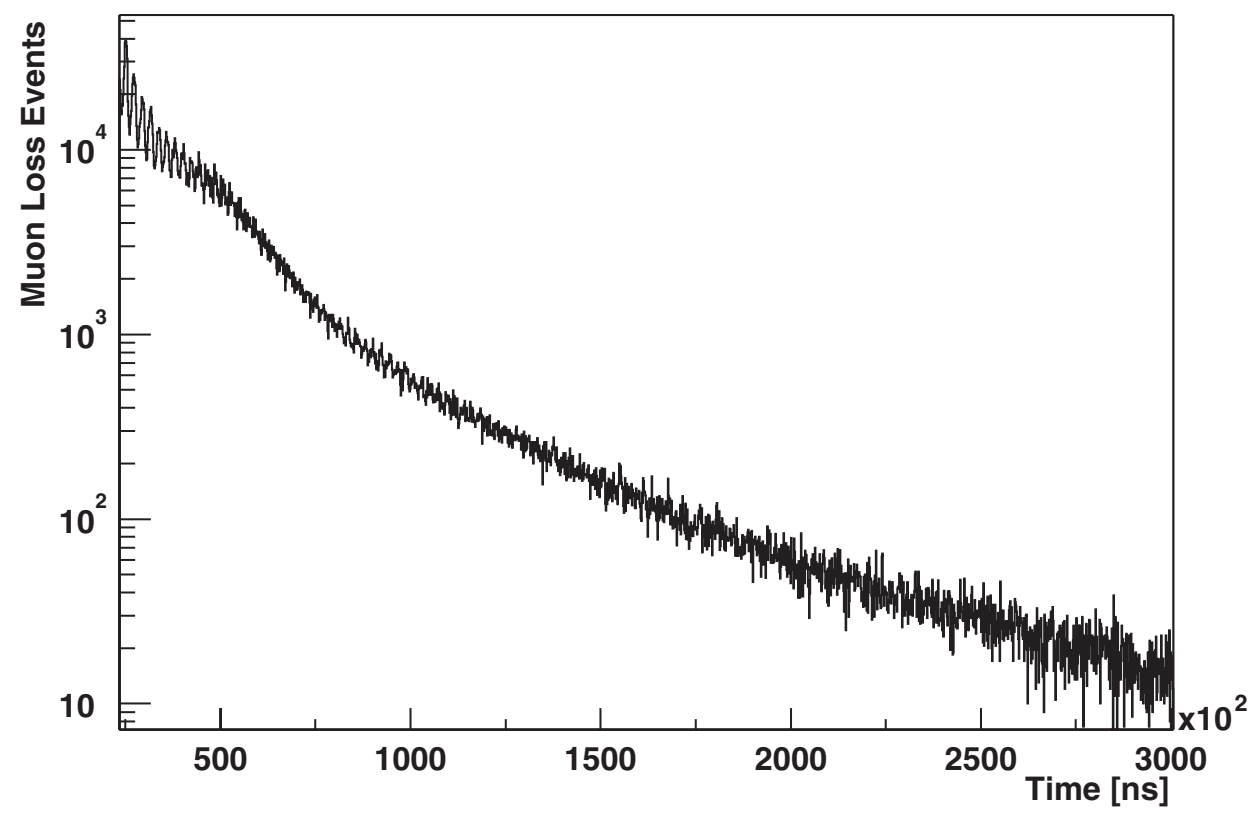

FIG. 35. Muon-loss rate vs time from the R00 period. Three consecutive and coincident FSD station signals form the muonloss signal. The loss function $L(t)$ is proportional to this raw data plot.

the Monte Carlo simulation. With an estimated acceptance of a few percent results from the fits indicate an approximate fractional loss rate of $10^{-3}$ per lifetime.
FNAL - sample from recent running

\section{Triple-Coincidences per Decay Positron}

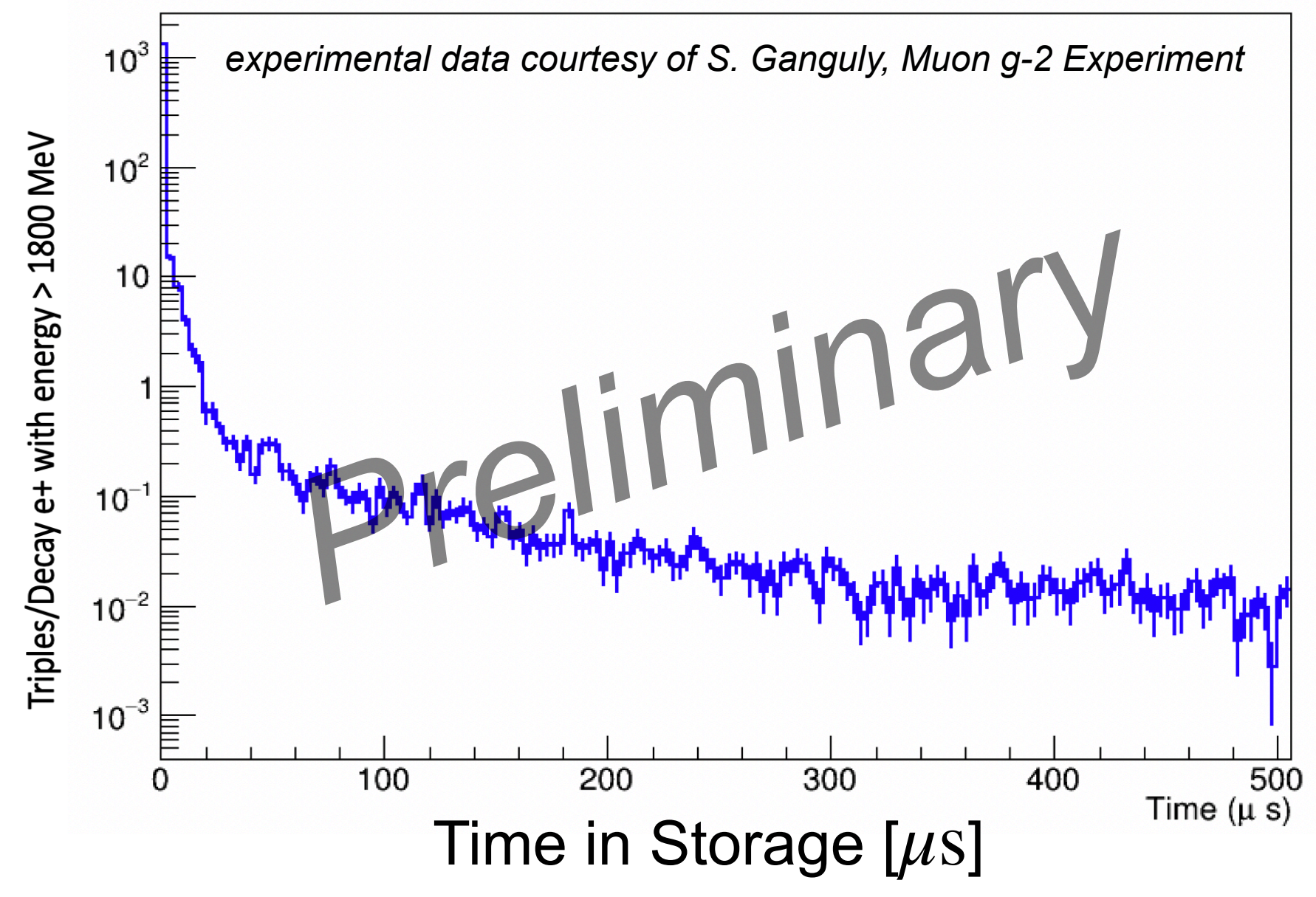




\section{Why the Concern?}

- Not a radiation concern; very low intensity, by storage ring standards; rather, it is important to understand in the final analysis of the experimental data

- wiggle plot and multi-parameter fit $\quad N(t)=N_{0} e^{-t / \tau_{\mu}}\left[1+A \cos \left(\omega_{a} t+\phi_{0}\right)\right]$

- if the contributions to the determination of the average initial phase evolves during the measurement, can give a systematic error on the actual precession frequency being sought

- Suppose muons which can reach the collimator (halo) have a different spin distribution than those of the central core:

» then, since only the halo particles get lost,

$$
\langle\phi\rangle=\frac{N_{c}\left\langle\phi_{c}\right\rangle+N_{h}\left\langle\phi_{h}\right\rangle}{N_{c}+N_{h}}
$$

can yield an
apparent precession: $\Delta \omega_{a}=\frac{d\langle\phi\rangle}{d t}=\left(\frac{N_{c}}{N_{c}+N_{h}}\right)\left(\frac{\dot{N}_{h}}{N_{c}+N_{h}}\right)\left[\left\langle\phi_{h}\right\rangle-\left\langle\phi_{c}\right\rangle\right]$

fraction in the core

loss

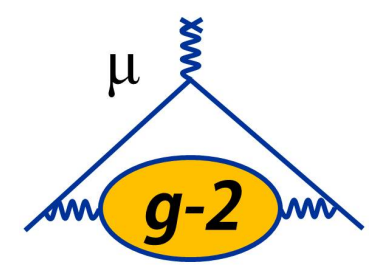




\section{Simple (but Detailed) Investigation}

- Consider this third loss mechanism - how long might it take some particles to finally encounter the collimator?

- Highly uniform fields in ring — basic linear theory describes motion well

- So, model injection; freeze phase space conditions, write to data frame

- Without tracking, perform this simple analysis:

- for each particle, calculate departure time and transverse position when lost

- from this information, create plot of intensity vs time; look at loss rates

- for particles lost during the measurement period, examine loss positions, momentum distributions, etc.

- compare computed loss rate and its evolution with time to actual data

- use 2-distribution model to understand level of the systematic effect 


\section{Model the Injection Process}

incoming from beam line: general characteristics confirmed by measurements
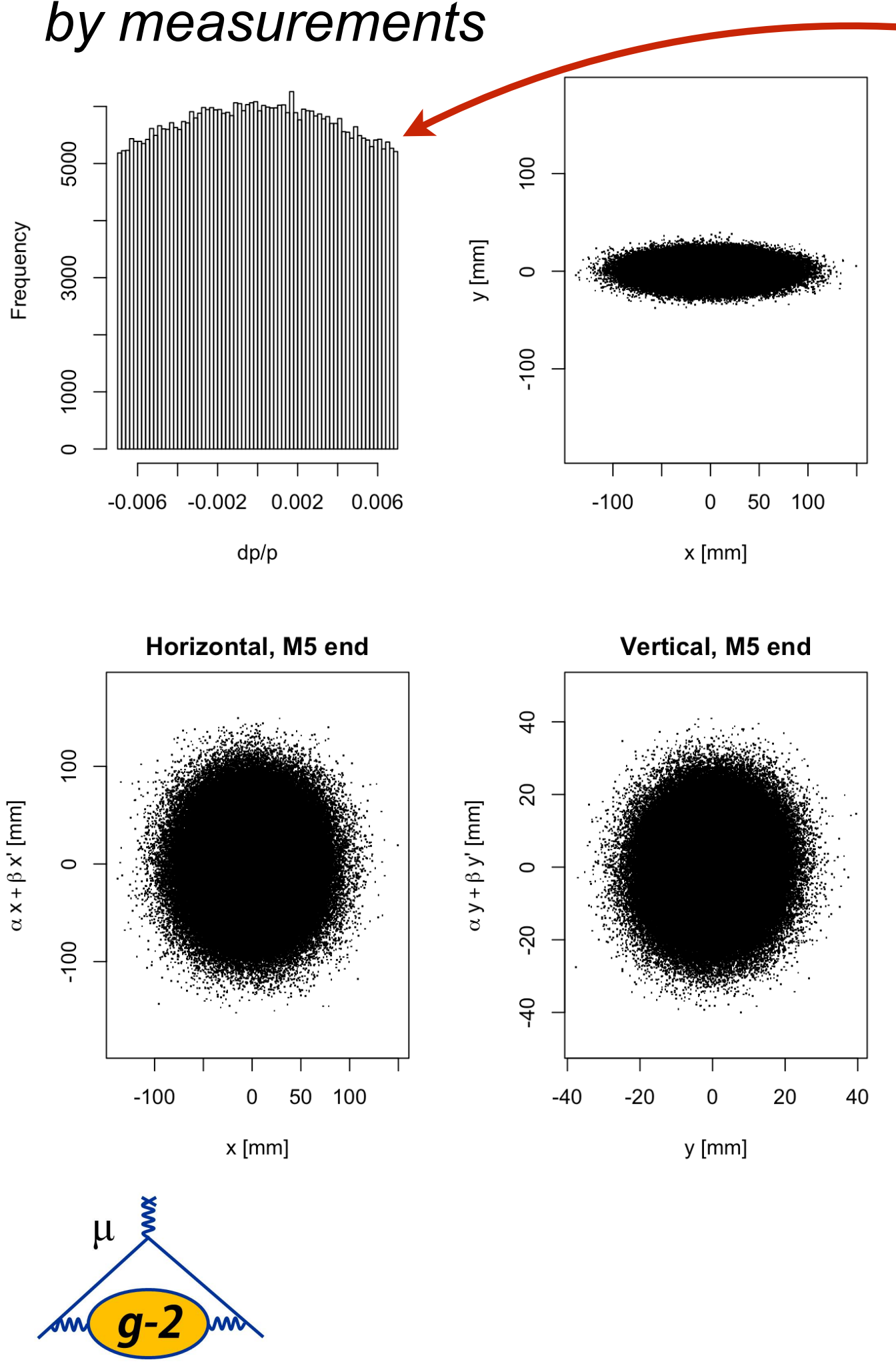

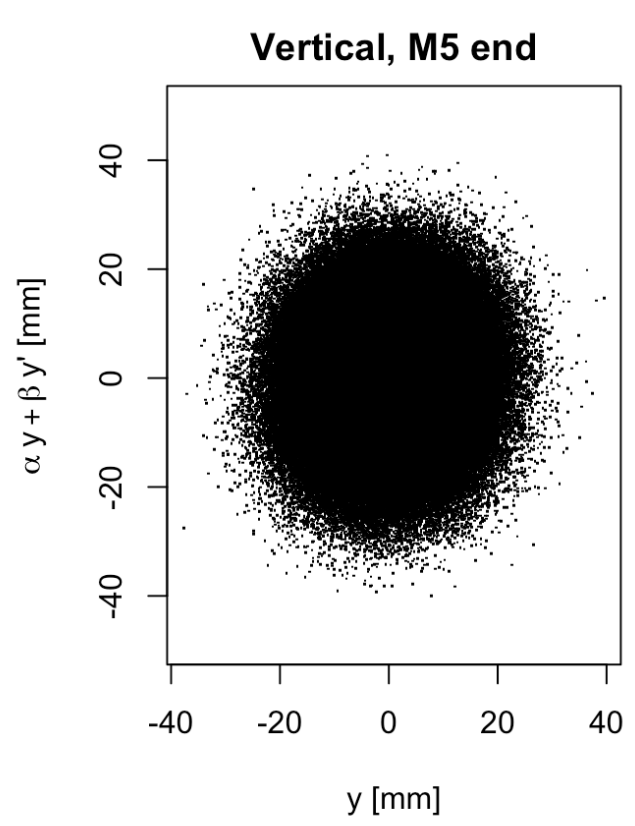

momentum spread of incoming beam is 10 times larger than ring acceptance

x phase space

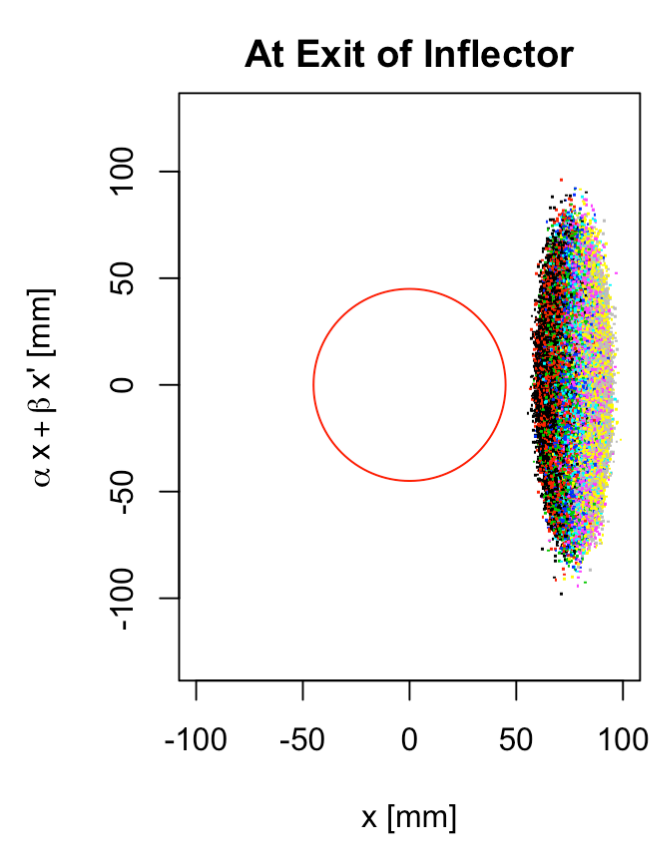

spreads out due to large dispersion mismatch

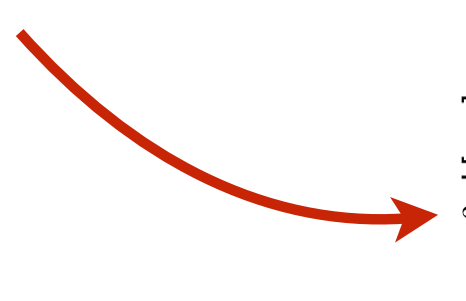

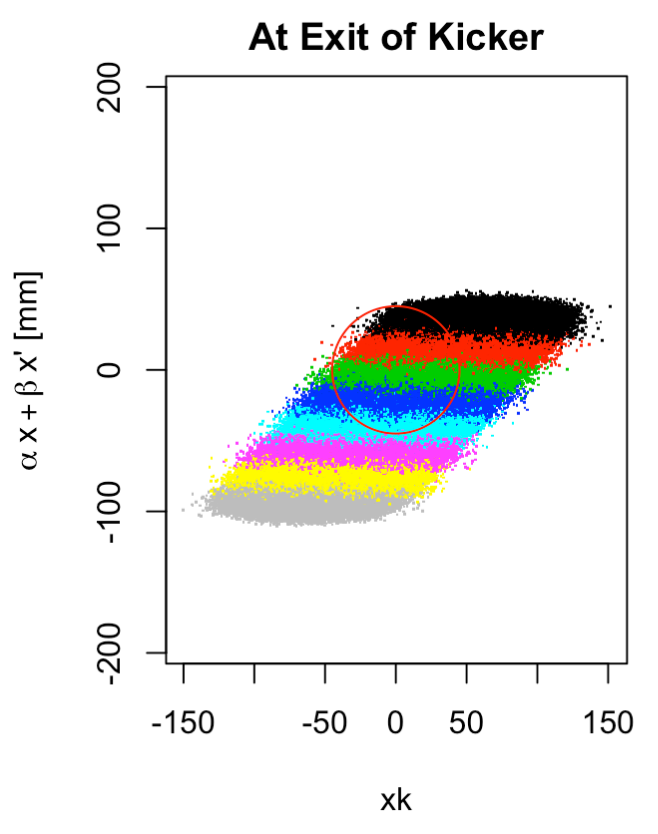

Northern Illinois University

\section{y phase space}
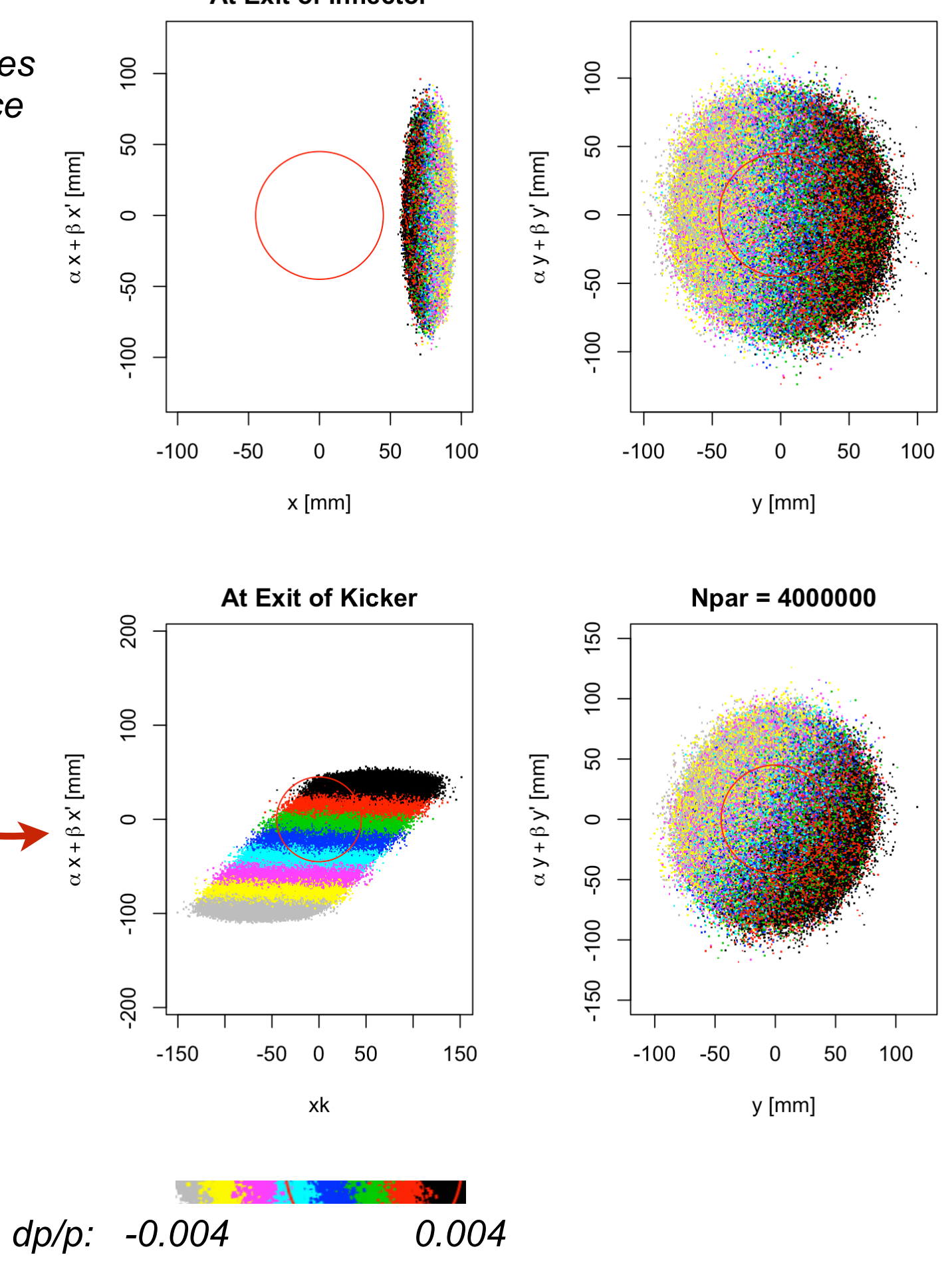


\section{Particle Storage}

- After modeling the injection kick, "freeze" the distribution at $n=0$

» Top/Left: $\quad x, y$ for all particles within $r=45 \mathrm{~mm}$

» Top/Right: momentum distribution

» Bottom/Left: $x$-phase space

»Bottom/Right: $y$-phase space

$$
\begin{aligned}
& x=D \cdot \delta+a \cos \left(2 \pi \nu_{x} n+\phi_{x}\right) \\
& \beta_{x} x^{\prime}+\alpha_{x} x=\quad-a \sin \left(2 \pi \nu_{x} n+\phi_{x}\right) \\
& y=\quad b \cos \left(2 \pi \nu_{y} n+\phi_{y}\right) \\
& \beta_{y} y^{\prime}+\alpha_{y} y=\quad-b \sin \left(2 \pi \nu_{y} n+\phi_{y}\right)
\end{aligned}
$$

compute

amplitudes,

initial betatron

phases

compile data

frame of info

for each particle

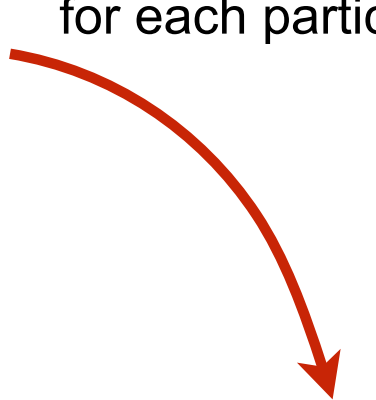

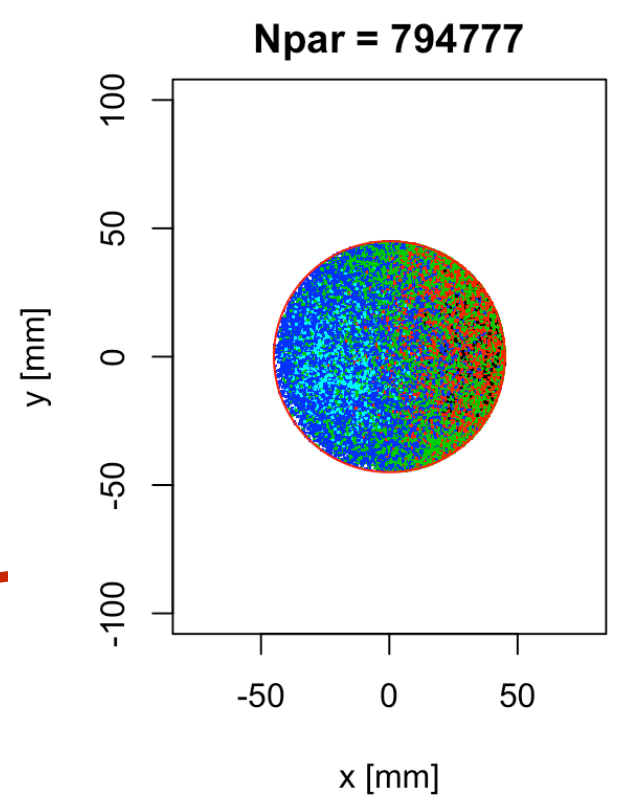

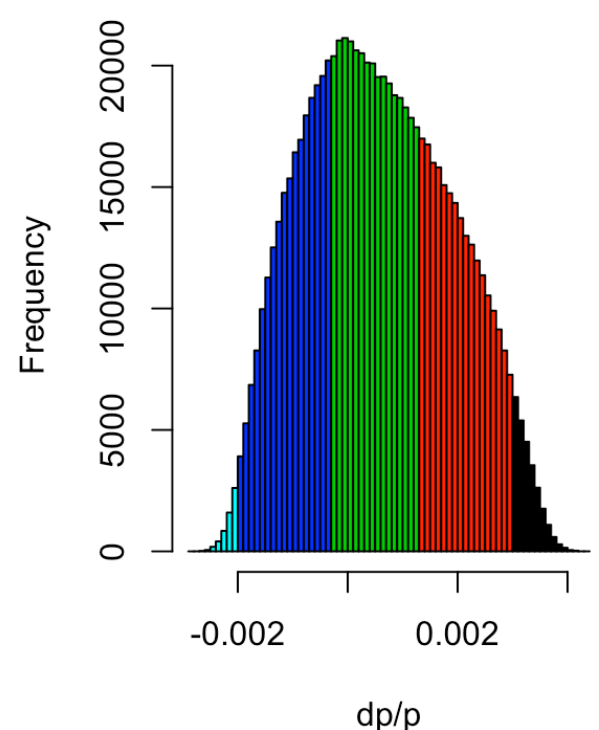

Northern Illinois University

\begin{tabular}{|l}
\hline \\
\hline 2 \\
\hline 3 \\
\hline$\vdots$
\end{tabular}

PX

y

Py
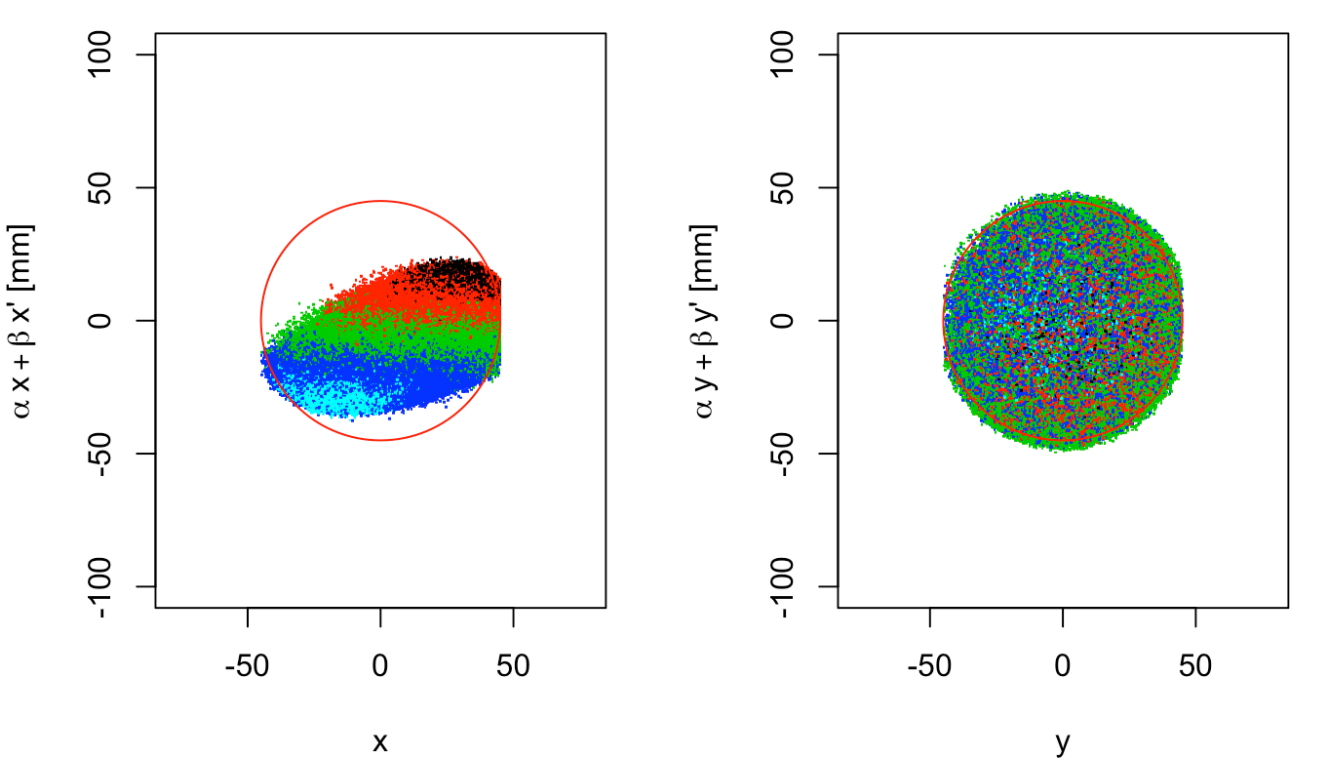

X

\begin{tabular}{l|l|l|l|l|l|l|l|l|l}
6.689066 & -72.42946 & -16.711137 & 18.62327 & -0.006698057 & 94.65789 & 25.02175 & -0.8713067 & 2.302132
\end{tabular}

\begin{tabular}{ll|l|l|l|l|l|l|l|l|l|l|l|l|l|}
6.689066 & -72.42946 & -16.711137 & 18.62327 & -0.006698057 & 94.65789 & 25.02175 & -0.8713067 & 2.302132 \\
\hline
\end{tabular}

ax

ay

phix

phiy

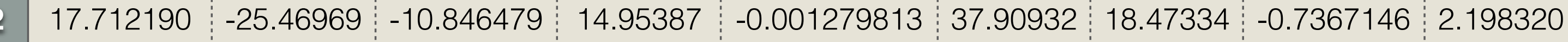

\begin{tabular}{|l|l|l|l|l|l|l|l|l|l|l|l|l}
5.610937 & -49.93999 & -31.715068 & -37.71116 & -0.003976070 & 62.64292 & 49.27451 & -0.9226708 & 4.013143 \\
\hline
\end{tabular}




\section{Times of Departure}

- For each particle, determine if its betatron amplitudes are such that the particle can reach the collimator:

$$
r^{2}(n)=\left[D \delta+a \cos \left(2 \pi \nu_{x} n+\phi_{x}\right)\right]^{2}+\left[b \cos \left(2 \pi \nu_{y} n+\phi_{y}\right)\right]^{2}
$$
» if not, then "Core" particle
$(\hat{r}<45 \mathrm{~mm})$
» if it can, then "Edge" particle
$(\hat{r} \geq 45 \mathrm{~mm})$

- For every Edge particle, compute at what value of $n$ will it reach the collimator and $(x, y)$ lost, and add these values to the particle data frame Results:
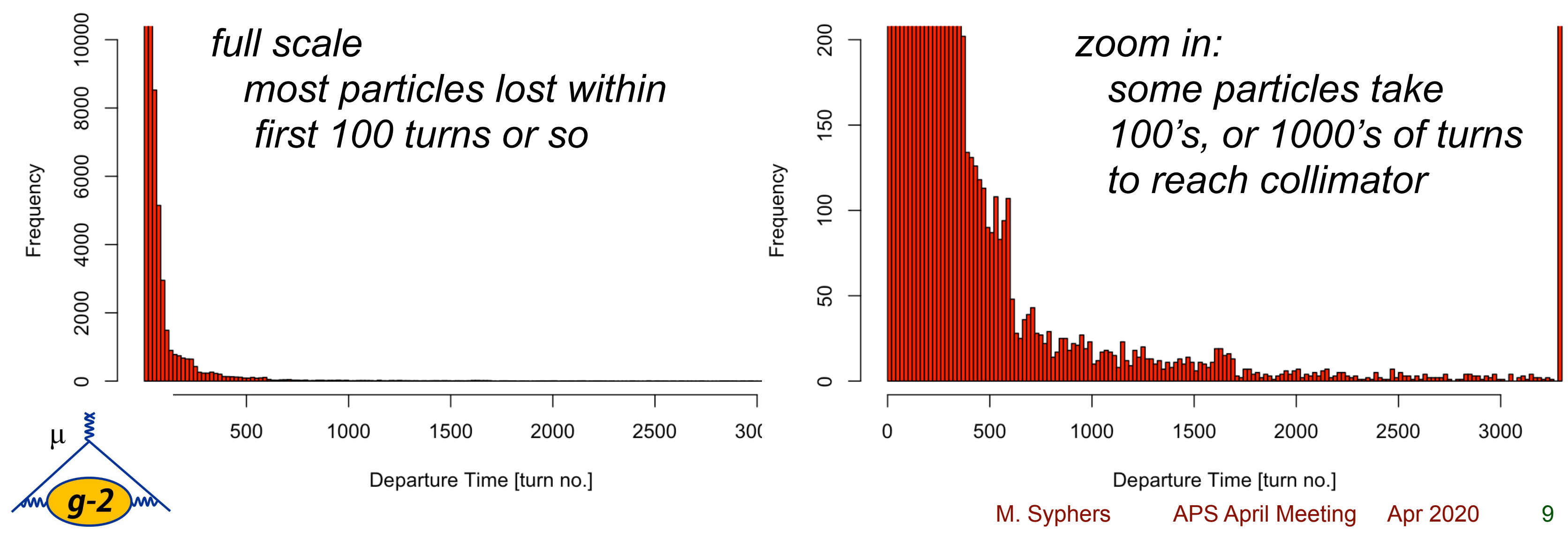


\section{Loss Patterns and Rates}

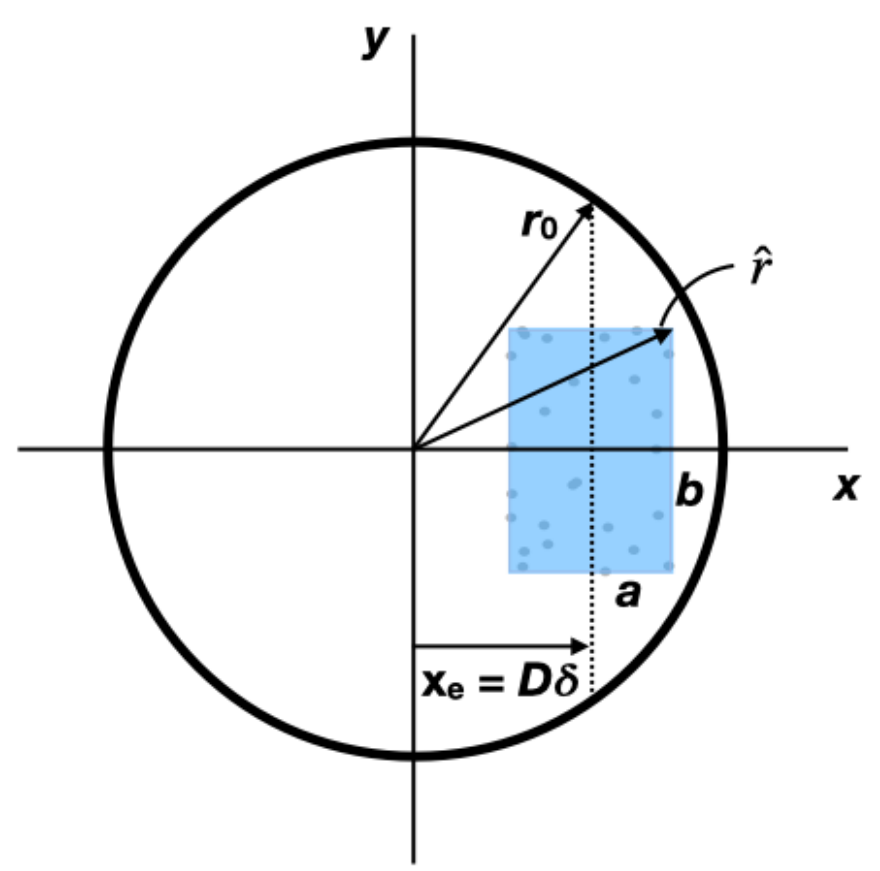

12

Northern Illinois University

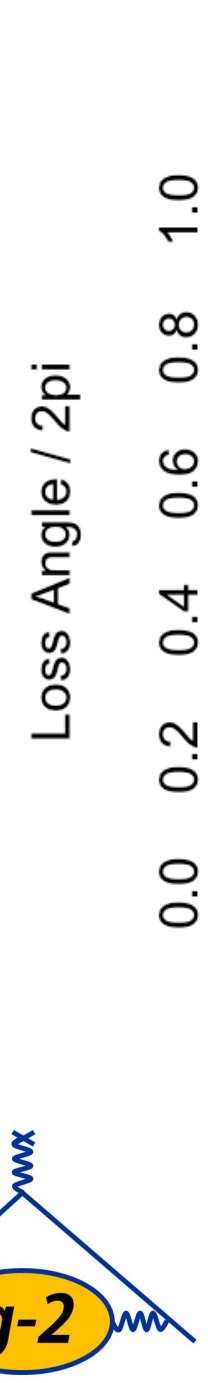

Angle $=0$ at 12 o'clock location

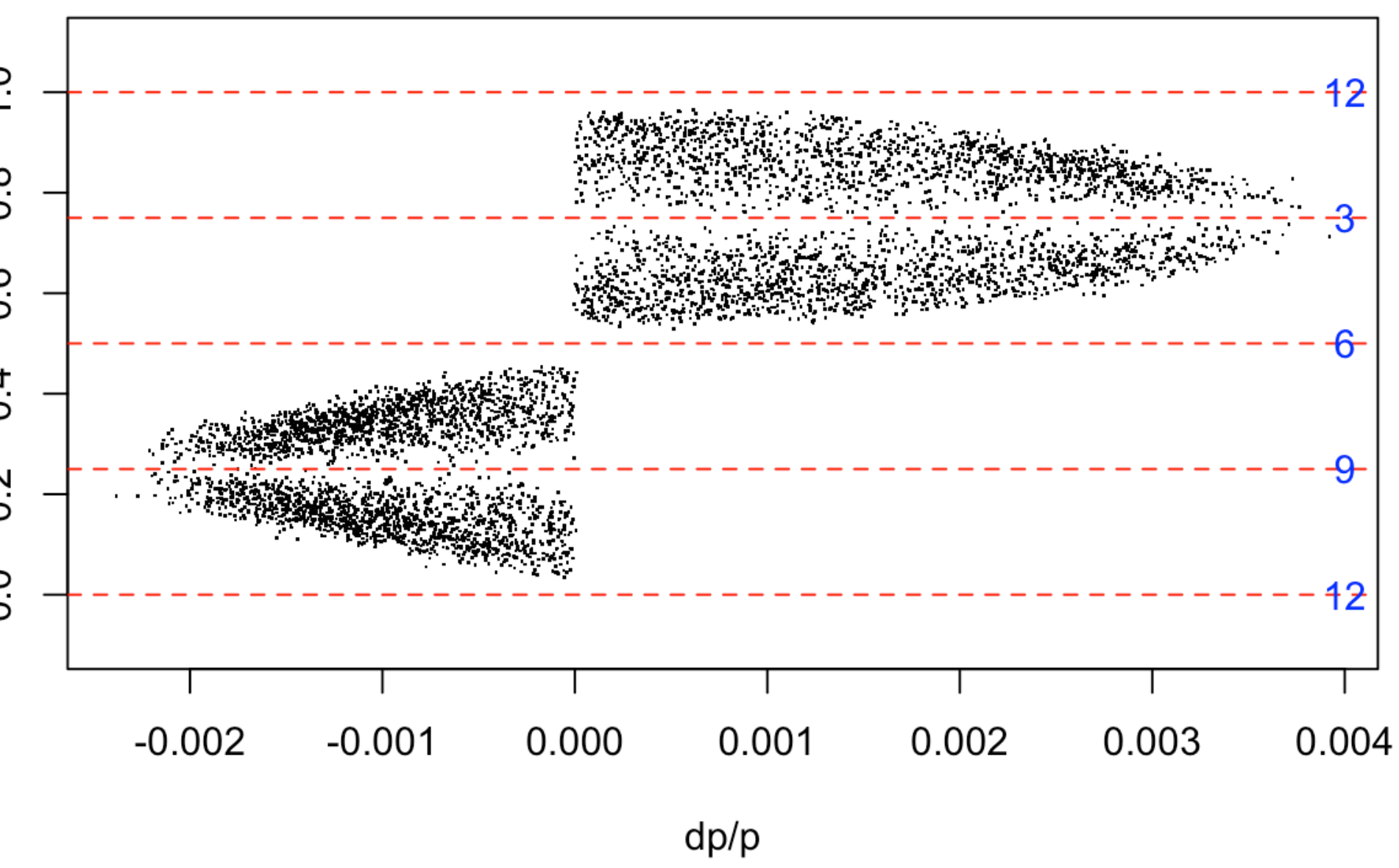

high momentum lost on right, low momentum lost on left 


\section{Loss Distributions on the Edge}

Northern Illinois University

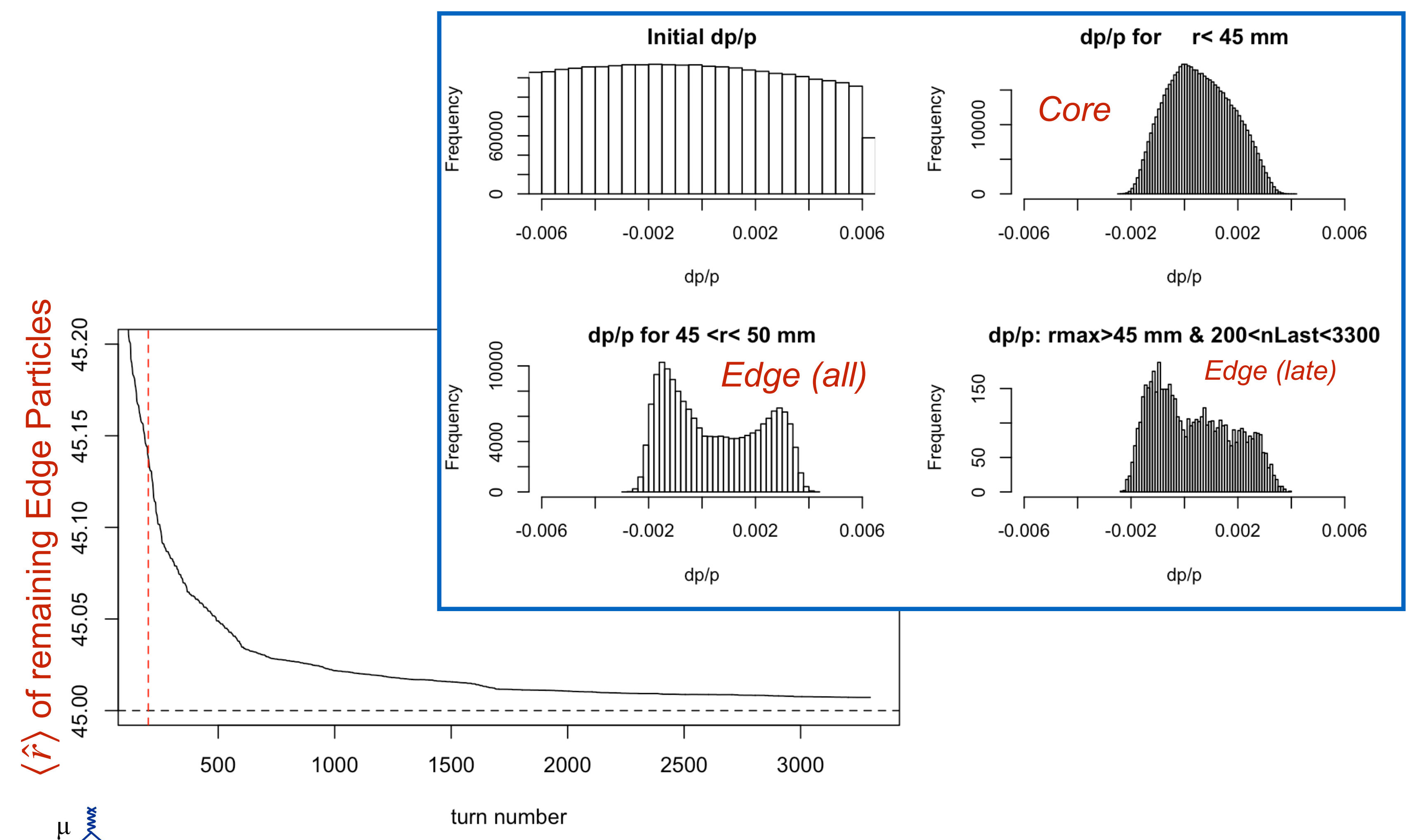



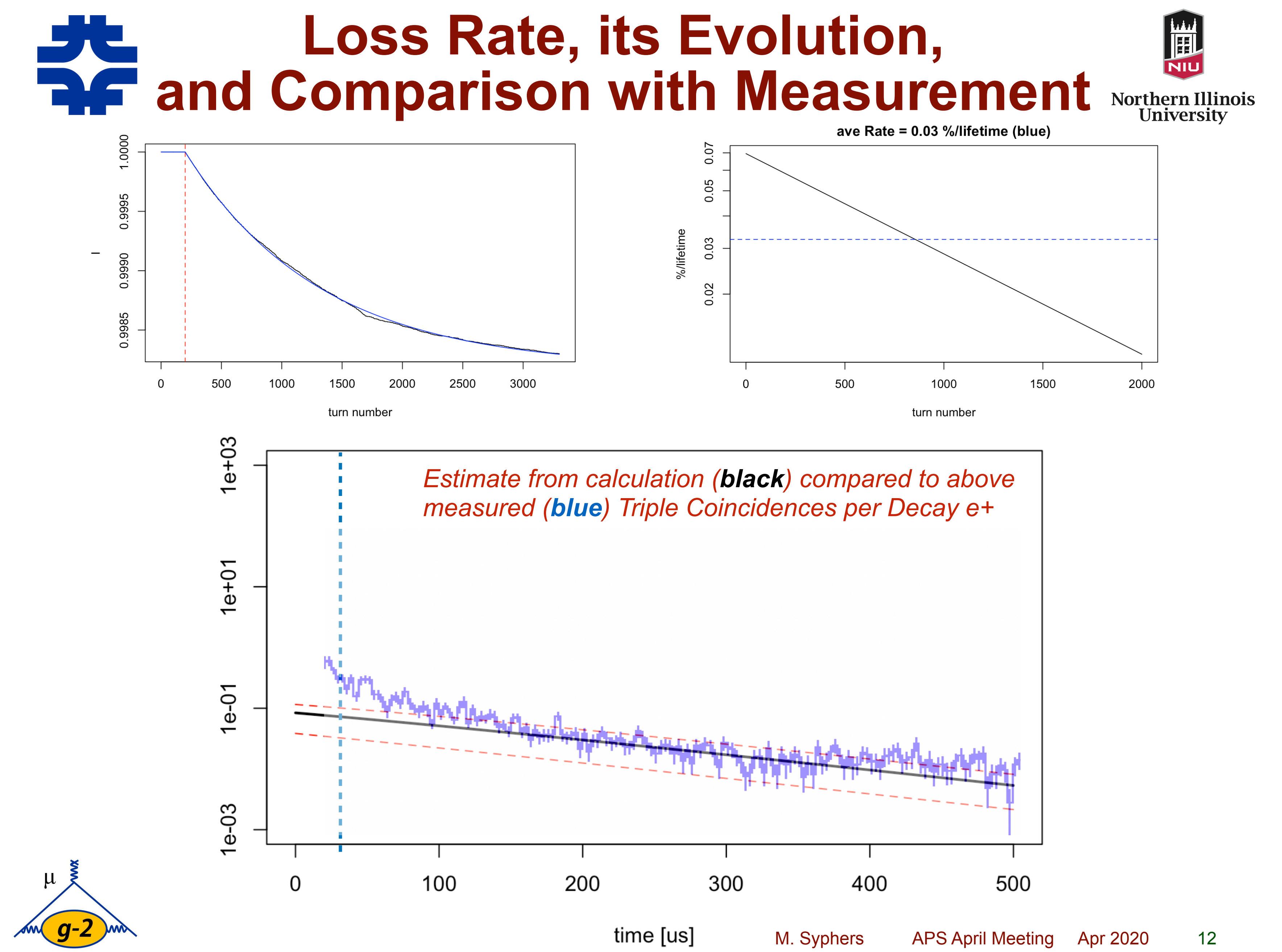

Estimate from calculation (black) compared to above measured (b/ue) Triple Coincidences per Decay e+

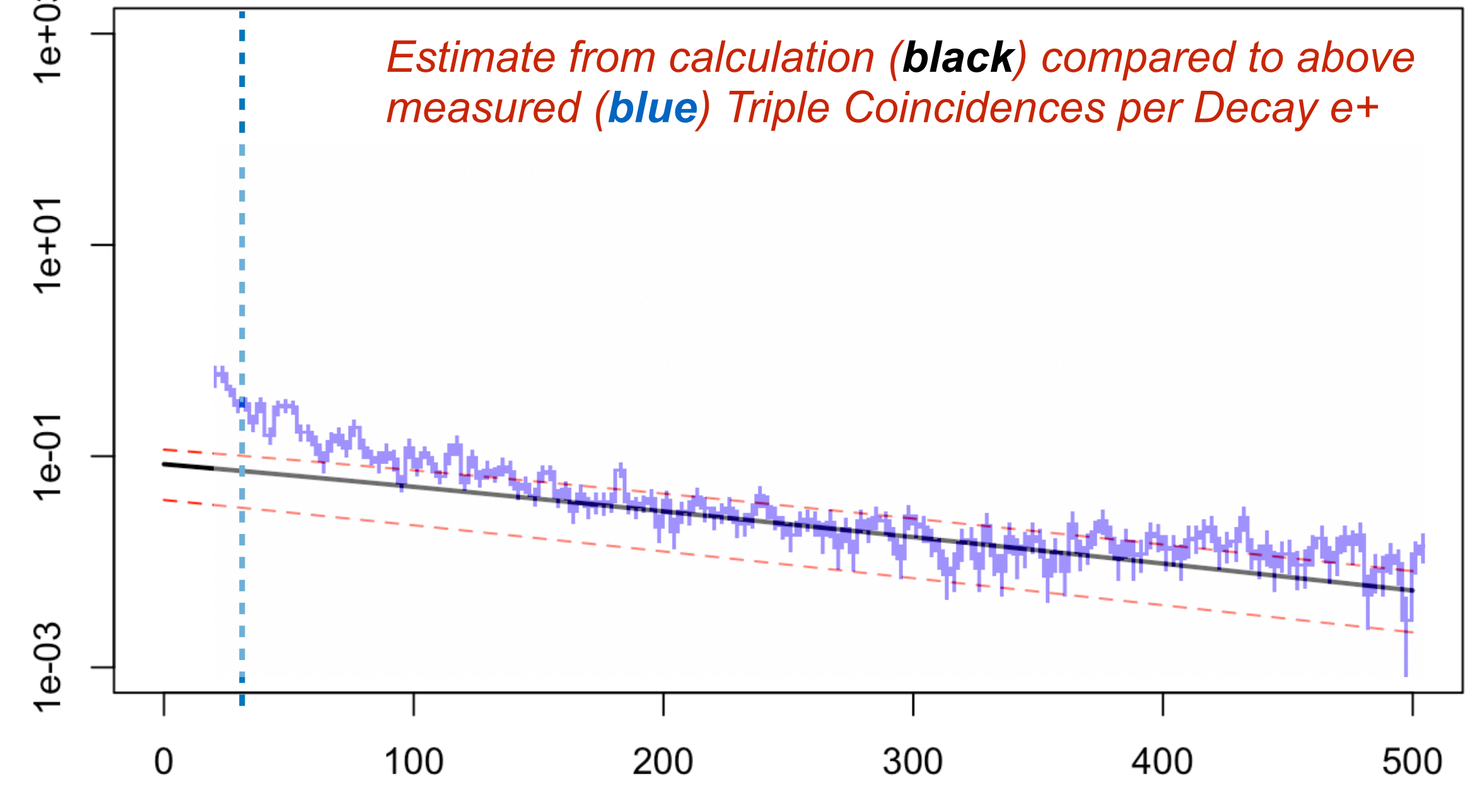

time [us] 


\section{Concluding Remarks}

- Data-frame-approach analysis; no standard-style particle tracking was involved. Expedient calculations from realistic phase space information

- Identified primary long-term loss mechanism

- incoming distribution "fills" available phase space

- two distinct distributions: Core particles and Edge particles

- some Edge particles may survive hundreds or thousands of revolutions

- Horizontal/vertical betatron oscillations yield largest transverse displacement when particle reaches appropriate "corner"; hence, most losses occur at the $45^{\circ}$ points on collimator perimeter

- Loss rates should be on the order of $0.01-0.1 \%$ per muon lifetime, and decrease exponentially with a time constant on the scale of 100-200 $\mu \mathrm{s}$

- both level and the exponential time constant are in line with observations

- Core/Edge distributions have different forms, but central values are similar and the loss rate is low enough that error on measurement of $\omega_{a}$ should be $\sim 10-20$ ppb level.

- Special thanks to H. Binney, J. Crnkovic, N. Froemming, S. Ganguly, D. Hertzog, and W. Morse for their help providing data, insightful comments, questions, and suggestions 